\title{
Simultaneous microscopic measurements of thermal and spectroscopic fields of a phase change material
}

\author{
M. Romano ${ }^{\mathrm{a}, *}$, M. Ryu ${ }^{\mathrm{b}}$, J. Morikawa ${ }^{\mathrm{b}}$, J.C. Batsale ${ }^{\mathrm{a}}$, C. Pradere ${ }^{\mathrm{a}, * *}$ \\ ${ }^{a}$ I2M, Département TREFLE, UMR CNRS 5295 - site ENSAM Esplanade des Arts et Métiers, 33405 Talence Cedex, France \\ ${ }^{b}$ Tokyo Institute of Technology, Department of Organic and Polymeric Materials, Tokyo 152-8552, Japan
}

\begin{abstract}
In this paper, simultaneous microscopic measurements of thermal and spectroscopic fields of a paraffin wax $n$-alkane phase change material are reported. Measurements collected using an original set-up are presented and discussed with emphasis on the ability to perform simultaneous characterization of the system when the proposed imaging process is used. Finally, this work reveals that the infrared wavelength contains two sets of important information. Furthermore, this versatile and flexible technique is well adapted to characterize many systems in which the mass and heat transfers effects are coupled.
\end{abstract}

Keywords: Infrared spectroscopy, Infrared thermography, Multispectral imaging, Phase change

\section{Introduction}

In chemical engineering, many processes are associated with simultaneous phenomena in which the temperature, concentration or pressure competitively drive the dynamics of the system, such as thermodiffusion, which is the phenomenon of a concentration gradient caused by a temperature gradient [1]. Inside reactive systems, the diffusion process is generated by the advancement of the reaction, which involves a concentration gradient and energy exchanges $[2,3]$. No reactive systems such as phase change materials can efficiently store thermal energy by absorbing/releasing high latent heat during the melting/solidifying process, which involves the modification (rupture or institution) of interatomic or intermolecular bonds [4, 5].

*e-mail: romano@enscbp.fr

**e-mail: c.pradere@i2m.u-bordeaux1.fr, Tel. +33.5.56.84.56.26

Preprint submitted to Industrial Engineering Chemistry Research
Simultaneous mass and energy exchanges are often associated with temperature variations and require special experimental measurement techniques to solve the overall balance equations [6].

The characterization of such systems is typically performed by probes or sensors that are intrusive. Additionally, sensors for intensives properties such as concentration and temperature do not have the same precision and resolution, and this can complicate the interpretation when the results are inconsistent. . Noninvasive techniques based on imaging or optics are emerging technologies that have recently been applied for the characterization of complex systems, such as for gas concentration measurements in a gas-solid fluidized bed [7] and for the measure- 
ment of the diffusion coefficients of liquid mixtures using the optical digital interferometry technique [8]. The imaging technique was used to perform three dimensional measurements using the spatial rotation of the samples in the near-infrared domain, and tomography was used to observe the dynamic behavior of flowing gases on a packed bed reactor [9]. In the terahertz domain, tomography was used to reconstruct the bone structure of the body [10].

Spectroscopy imaging techniques analyze chemical systems via the identification of the molecular composition, which can be related to the concentration after applying a calibration protocol. This characterization is typically achieved using analytical spectroscopy methods. Most of these techniques have proved to be well suited for quantitative measurements. Moreover, the techniques characterize the surface of a sample via mapping (a single element detector is scanned over the sample surface). This scanning technique is difficult to use due to problems associated with transient characteristics. As a result, considerable efforts have been made in the past few years to develop multispectral imaging instruments

In this paper, an infrared camera, monochromator and optics were combined to achieve fine simultaneous microscopic measurements of the thermal and spectroscopic fields of a paraffin wax $n$-alkane phase change material. The originality of this work lies in the analysis of phase change materials, which are of great interest for many applications, at different temperatures using a novel and well-adapted technique. Here, after describing the experimental set-up and micro-scale measurements in the transmission mode, the processing method, which is based on the simultaneous acquisition of two images, is presented. The characterization results of the phase change material are reported and discussed. This work reveals that by using well-adapted instrumentation, it is possible to decrypt two pieces of important information contained in the infrared wave- length. Furthermore, this versatile and flexible technique is well adapted to characterize many systems in which the chemical properties and temperature effects are coupled.

\section{Materials and methods}

\subsection{Experimental set-up}

The imaging instrument presented in this study is shown in Figure 1, and it is composed of a high-speed infrared (IR) camera (Phoenix Indigo) and a grating monochromator to select the excitation wavelength (CT-10, JASCO Corporation, Tokyo, Japan).

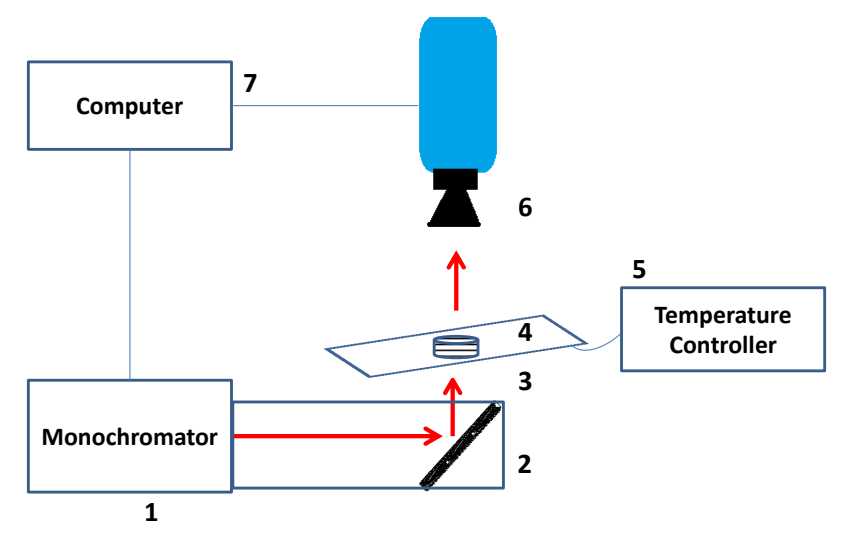

Figure 1: Scheme of the experimental set-up in horizontal transmission mode: 1) Monochromator, 2) Parabolic mirror, 3) Microscope heat stage and sample holder, 4) Sample, 5) Temperature controller, 6) Indigo Infrared InSb cooled camera, and 7) Computer for data acquisition

The beam emitted from the slit (output of the monochromator, Figure 1-1) is monochromatic. The source light is transmitted from the monochromator to a parabolic mirror (Figure 1-2) to guide it through the sample (Figure 1-4); the transmitted light is recorded by the IR camera used as a sensor (Figure 1-6) to perform the micro-scale thermography. The camera has an indium-antimony ( $\mathrm{InSb}$ ) detector composed of a focal plane array (FPA) featuring 81,920 pixels (matrix $320 \times 256$ ) that act as independent sensors, and the simultaneous measurement of 81,920 spots can be performed using the snapshot mode in 1 $\mu$ s with an optimum wavelength operating between 3 and $5 \mu \mathrm{m}$. 
The camera is equipped with an original designed microscopic lens (from Tokyo Tech Institute, Japan) with a magnification of $7.5 \mathrm{X}$ and a spatial resolution of $4.1 \mu \mathrm{m} \mathrm{x} 4.1 \mu \mathrm{m}$ (at 5 microns, the spatial resolution was tested with a standard USAF 1951 micro-scale, [11]). The frame rate acquisition was set to $60 \mathrm{~Hz}$. The photon count of the emitted intensity was detected with 16 bit sensitivity. The infrared spectra were acquired using the synchronization of the monochromator and the IR camera with homemade LabVIEW software to control the acquisition frequency and to scan the wavelength over the domain of interest. The same software also facilitates the frame recording and data saving in appropriate formats for post-processing. A calibration protocol was used for the alignment of the optical set-up and for the lamps (ceramic heater). For ensuring the transmission of the wavelengths, some reference standard materials were used to calibrate the proposed instrument. The sample holder shown in Figure 1-3 is a S84 microscope heat stage (PIKE technologies) with two sapphire windows at the top and bottom of the device to enable the observation in transmission mode, and inside the enclosure, the temperature is controlled with $\pm 0.1{ }^{\circ} \mathrm{C}$ precision.

\subsection{Samples and measurement process}

In this study, $n$-alkane icosane (average molar mass $=272$ g. $\mathrm{mol}^{-1}$ ) (Tokyo Chemical Industry, Co. Ltd.) was chosen to study the solid to liquid phase transition in the temperature range from $22{ }^{\circ} \mathrm{C}$ to $48{ }^{\circ} \mathrm{C}$ in $4{ }^{\circ} \mathrm{C}$ steps, as shown in Figure 2. The paraffin wax was deposited between two thin sapphire circular plates (diameter of $10 \mathrm{~mm}$ and thickness of approximately $100 \mu \mathrm{m}$ ), and the thickness of the specimen film was less than $10 \mu \mathrm{m}$. First, the spectrum of the sapphire plates was acquired in the spectral range from 2.6 to $5.2 \mu \mathrm{m}(\Delta \lambda=26 \mathrm{~nm})$ as a function of the temperature range. When the imposed temperature reached the plateau (stabilization time of $3 \mathrm{~min}$ ), the signal was recorded, as shown in Figure 2. Then, both the tem- perature (proper emission of the sample) and the spectroscopic measurements were performed over the spectral range and for the same temperature range as in the double sapphire measurement. In this study, the transient state that occurred during the phase change was not recorded. Only the steady states (plateau of each step during the temperature ramp) of the solid and liquid phases are reported.

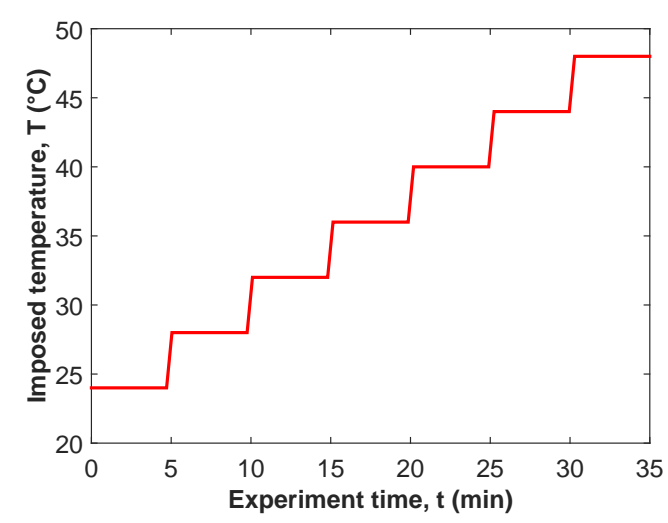

Figure 2: Scheme of the heating process: the temperature imposed on the sample is measured by the thermal probe inside the S 84 microscope heat stage.

\subsection{Spectral acquisition}

The imaging instrument schematized in Figure 1 has been called FIIST (Fast Infrared Imaging Spectroscopy Technique, [12]). A FPA and a microscope were used to generate the imaging area of $1.5 \mathrm{~mm} \times 1.5 \mathrm{~mm}$, as depicted in Figure 3.A. The infrared image of the sample $\left(\mathrm{C}_{20} \mathrm{H}_{42}\right.$ specimen between two sapphire thin plates at $40^{\circ} \mathrm{C}$ ) was taken at a wavelength of 3.84 $\mu \mathrm{m}$. The same image was generated using the spectral imaging instrument, Perkin ELMER spotlight (spatial resolution of 6.25 $\mu \mathrm{m}^{2}$ ), as depicted in Figure 3.B. The main difference between both images is the acquisition time ( 3 minutes (FIIST) $\lll 15$ minutes (ELMER), considering the lowest acquisition time for both techniques) using FIIST. For each wavelength, one image or a sequence of images (snapshot) can be obtained to improve the signal-to-noise ratio. Considering the characteristics of the sample surface, every pixel of the image contains the spectral 
response function of the wavelength. In contrast, the Perkin Elmer imaging spectrometer (equipped with a linear array MCT detector) operates by scanning the surface, which makes the acquisition slower even if the Fourier transform spectral acquisition is faster. However, the FIIST spectral acquisition is limited by the mechanical displacement of the monochromator from $2.6 \mu \mathrm{m}$ to $5.2 \mu \mathrm{m}$. Thus, the overall acquisition of the spectra remains fast and full of spectral information «big data $\gg$ issues containing $\approx 150$ million data points for the acquisition of a simple spectrum. The details concerning the sensitivity and the performances of the technique were previously reported [12].

A
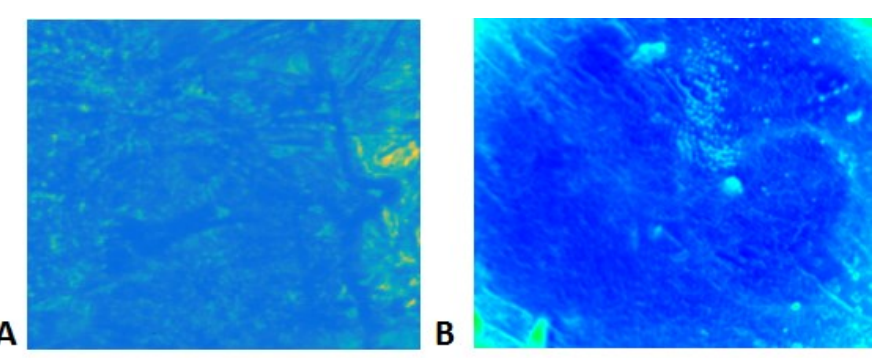

Figure 3: A. Spectroscopic image acquired using the previously described experimental set-up, FIIST, at a wavelength of $3.84 \mu \mathrm{m}$. B. Spectroscopic image acquired using the spectral imaging instrument Perkin ELMER spotlight at 3.84 $\mu \mathrm{m}$.

\subsection{Processing method}

The acquisition frequency of the IR camera was synchronized with the mechanical chopper at the slit (output) of the monochromator (Figure 1-1). This configuration enables the recording of two different images: one containing the thermal and the spectroscopic information (when the chopper is open) and the other containing only the thermal information (proper emission of the sample when the chopper is closed), as shown in Figure 4. Both images are recorded and transferred to the computer using a frame grabber. In-house software written in LabVIEW and Matlab was used to control the frequency and to scan the wavelength domain of interest. The same software also facilitated the frame grabbing and data recording in the appro- priate formats for subsequent analysis, based on the two-image methodology.

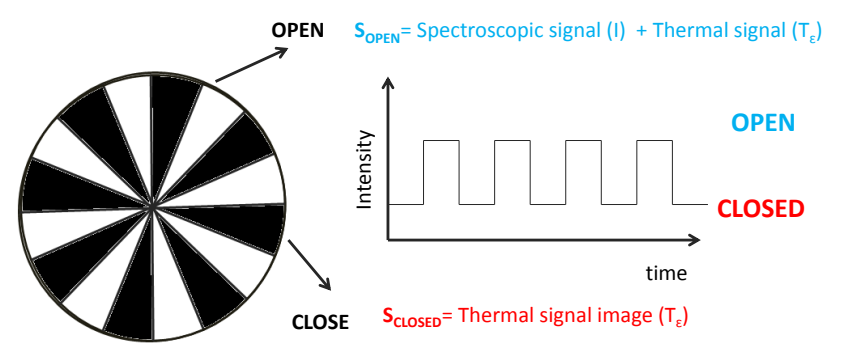

Figure 4: A mechanical chopper placed inside the monochromator is used to acquire the two different images. When the chopper is closed, the light source from the spectrometer is cut, and the recorded images of the sample contain only the thermal information (proper emission of the thermal scene) measured by the detector of the camera $\left(I_{\text {closed }}\right)$. When the chopper is open, the light source from the spectrometer is transmitted through the sample to the detector of the IR camera; thus, the recorded images $\left(I_{\text {open }}\right)$ contain the thermal information $(\mathrm{T})$ plus the spectroscopic signal $(\mathrm{S})$.

Based on this processing method, the notation is summarized as follows.

\begin{tabular}{|ll|}
\hline & Nomenclature \\
$\mathrm{x}, \mathrm{y}$ & Spatial distribution $(\mu \mathrm{m})$ \\
$\lambda$ & Wavelength $(\mu \mathrm{m})$ \\
$\mathrm{S}$ & Raw measured signal (DL, digital level) \\
$\mathrm{T}_{\epsilon}$ & Temperature signal: proper emission (DL, digital level) \\
$\mathrm{I}$ & Transmission spectroscopic signal (DL, digital level) \\
\hline
\end{tabular}

Based on the previous notations, the raw signal of the image acquired when the chopper is open is defined as:

$$
S(x, y, \lambda, T)=T_{\epsilon}(x, y)+I(x, y, \lambda, T)
$$

where $\mathrm{T}$ specifies a given temperature from $24^{\circ} \mathrm{C}$ to $48^{\circ} \mathrm{C}$. In contrast, when the chopper is closed, the image contains only the proper emission spatial distribution, which is defined as $T_{\epsilon}(x, y)=\epsilon \sigma s\left(T(x, y)^{4}-T_{0}^{4}\right)$, where the emissivity $(\epsilon)$ is a dimensionless unit between 0 and 1 , the Stefan-Boltzmann constant is $\sigma=5.670373 \times 10^{-8} W \cdot m^{-2} \cdot K^{-4}$, the observed surface $(s)$ of the sample is $m^{2}$, the imposed temperature is $\mathrm{T}(\mathrm{x}, \mathrm{y})$ and the initial temperature is $T_{0}$. The energy measured $\left(T_{\epsilon}(x, y)\right)$ by the IR camera in arbitrary units is called the Digital Level (DL), and it is related to the energy units (Watt) or degrees $\left({ }^{\circ} \mathrm{C}\right.$ ) after applying a calibration curve (shown in the results section). 
To obtain an image containing only the spectroscopic information in transmission mode, the following relation is applied:

$$
I(x, y, \lambda, T)=S(x, y, \lambda, T)-T_{\epsilon}(x, y)
$$

This is equal to the subtraction of the image containing the thermal signal from the one containing both signals. For this study, the spectroscopic signal of the 2 sapphire plates used $\left(I_{s a}\right)$ is defined as:

$$
I_{s a}(x, y, \lambda, T)=S_{s a}(x, y, \lambda, T)-T_{\epsilon, s a}(x, y)
$$

where the global spectroscopic signal $\left(I_{\text {sample }}\right)$ of both the sapphire plates and the sample (sandwich configuration) can be defined as follows:

$$
I_{\text {sample }}(x, y, \lambda, T)=S_{\text {sample }}(x, y, \lambda, T)-T_{\epsilon, \text { sample }}(x, y)
$$

The two previous relations, $I_{s a}$ and $I_{\text {sample, }}$ are applied to the images, and from them, we can calculate the transmittance spectrum of the sample, expressed as:

$$
I(x, y, \lambda, T)=\frac{I_{\text {sample }}(x, y, \lambda, T)}{I_{s a}(x, y, \lambda, T)}
$$

\section{Results and discussions}

\subsection{Spectra of the paraffin wax}

The $\mathrm{C}_{20} H_{42}$ was analyzed using the proposed techniques at different temperatures, and the intensity of the obtained spectral images was averaged along the $\mathrm{x}$ and $\mathrm{y}$ directions. The transmittance spectra of the sample as a function of the wavelength for several temperatures were obtained by applying equation 5 and are shown in Figure 5.

The $n$-alkane is composed of a chain of methylene units terminated at each end by methyl groups. The IR spectrum is expected to primarily contain the peaks attributed to the stretching

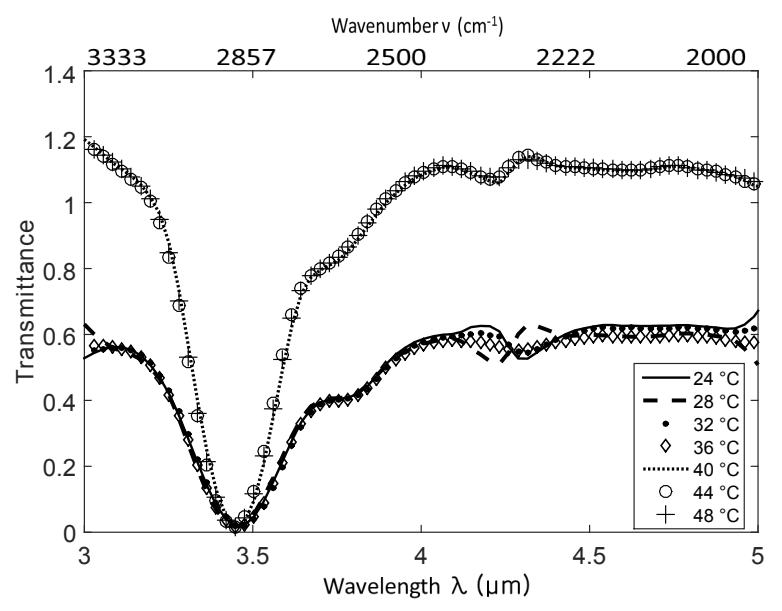

Figure 5: The transmittance intensity images obtained (applying equation 4, arbitrary units) were averaged along the $\mathrm{x}$ and $\mathrm{y}$ directions of the spectra of the sample at different temperatures of $24{ }^{\circ} \mathrm{C}$ (solid line), $28{ }^{\circ} \mathrm{C}(--), 32{ }^{\circ} \mathrm{C}(\bullet)$, $36{ }^{\circ} \mathrm{C}(\diamond), 40{ }^{\circ} \mathrm{C}$ (dotted line), $44{ }^{\circ} \mathrm{C}(\circ)$, and $48^{\circ} \mathrm{C}(+)$ over a spectral range of $3 \mu \mathrm{m}$ to $5 \mu \mathrm{m}$, which is equivalent to $3333 \mathrm{~cm}^{-1}$ to $2000 \mathrm{~cm}^{-1}$.

and bending vibrations of methylene. The spectrum obtained by the FIIST imaging spectrometer is shown in Figure 5 and is in good agreement with the infrared methylene bands that are observed and overlap. The two peaks dominate the spectra in the spectral region of interest: methylene stretching bands at 3.42 $\mu \mathrm{m}\left(2920 \mathrm{~cm}^{-1}\right)$ and $3.5 \mu \mathrm{m}\left(2850 \mathrm{~cm}^{-1}\right)$. In Figure 5, two different states are clearly identified: a solid state between $24{ }^{\circ} \mathrm{C}$ and $36{ }^{\circ} \mathrm{C}$ and a liquid state between $40{ }^{\circ} \mathrm{C}$ to $48^{\circ} \mathrm{C}$. The phase change occurs between $36{ }^{\circ} \mathrm{C}$ and $40^{\circ} \mathrm{C}$. Moreover, the paraffin exhibits a higher transmittance in the liquid state than in the solid state (transmittance ratio $\approx 2$ ). The transmittance in the liquid state is greater than 1 (see in figure 5 the spectra at $40{ }^{\circ} \mathrm{C}$ (dotted line), $44^{\circ} \mathrm{C}(\circ)$ and $48^{\circ} \mathrm{C}(+)$ ). This is because when the spectrum of the double sapphire superposed plates is obtained (reference spectrum), a part of the light does not pass through them until it reaches the detector. This does not mean that the medium absorbs this light but that a portion of the light is lost in the reflections taking place inside the empty superposed plates at the acquisition of the reference spectrum. 


\subsection{Spectroscopic imaging}

The sample characterization was performed at several temperatures, and in this section, the spectroscopic images are reported. Figure 6 shows the solid state $\mathrm{C}_{20} \mathrm{H}_{42}$ at $\mathrm{T}=24{ }^{\circ} \mathrm{C}$, and Figure 7 shows the liquid state at $\mathrm{T}=48{ }^{\circ} \mathrm{C}$. Both figures report four images at different wavelengths $(\lambda=3.14,3.42,3.84$ and $4.54 \mu \mathrm{m}$, spectra shown in figure 5). As previously mentioned, only the steady states of the solid and liquid state are reported. The side colorbar of the figures represents the transmittance. Thus, in Figure 6, the transmittance cartography can be observed. These data reveal that the transmittance of the sample varies slightly in a spatial manner, in contrast with the average value reported in Figure 5 at $24{ }^{\circ} \mathrm{C}$ (solid line). Some regions with higher or lower transmittance values can be perceived because of the thickness variation in this particular part of the deposited paraffin wax film. A solid structure of paraffin wax is observed in the images. In Figure 7, the liquid state is more homogenous than the solid paraffin wax film, which can be explained by the rearrangement after the melting of the phase change material. However, in both states (solid and liquid), for the characteristic peak of the paraffin $(3.4 \mu \mathrm{m}$, Figure 6 and 7.C), the transmission value is close to zero (absorption of the spectrum).

To analyze the global spectroscopy behavior as a function of the temperature, the integration of the spectroscopic images over the characteristic peak (cumulative addition of the image wavelengths, Gaussian distribution of around $3.4 \mu \mathrm{m}$ ) for each temperature was performed, and the resulting images are plotted in Figure 8.A. From the images, the transmittance was averaged along the $\mathrm{x}$ and $\mathrm{y}$ directions, and this value is plotted as a function of the imposed temperature in Figure 8.B. This last graph clearly shows that in the solid or liquid state, the $\mathrm{C}_{20} \mathrm{H}_{42}$ transmittance average value is constant, whereas the transmittance value is different for both states. The transmission in the

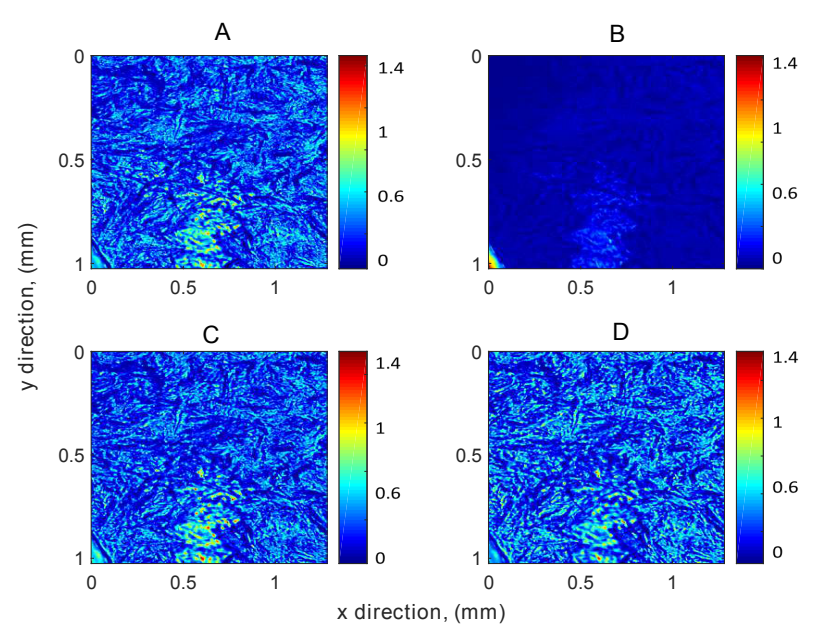

Figure 6: Spectroscopic images of the paraffin wax in the solid state $\left(24^{\circ} \mathrm{C}\right.$, before the phase change) for several wavelengths: A. $\lambda=3.14 \mu \mathrm{m} \mathrm{B} . \lambda=3.42$ $\mu \mathrm{m} \mathrm{C}$. $\lambda=3.84 \mu \mathrm{m}$ D. $\lambda=4.54 \mu \mathrm{m}$. The side colorbar in each image indicates the transmittance values.

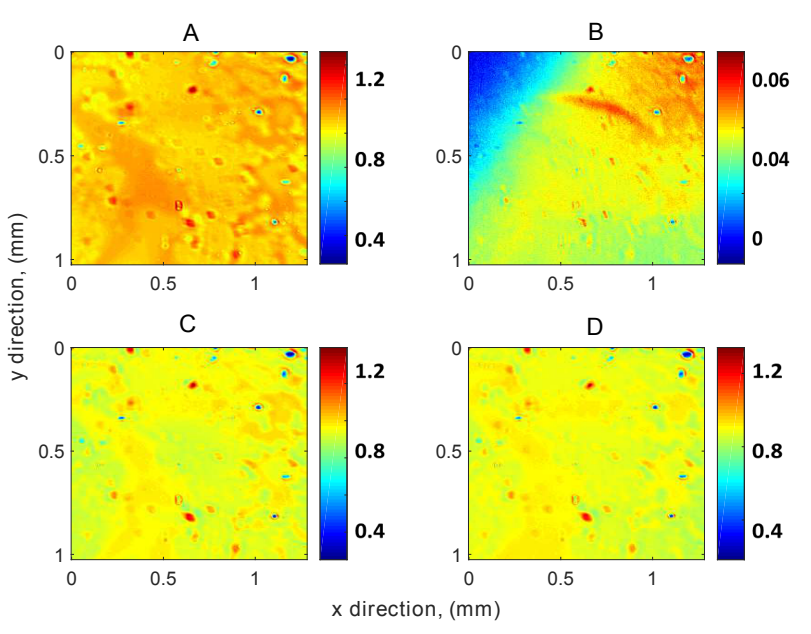

Figure 7: Spectroscopic images of the $\mathrm{C}_{20} \mathrm{H}_{42}$ in the liquid state $\left(48^{\circ} \mathrm{C}\right.$, after the phase change) for several wavelengths: A. $\lambda=3.14 \mu \mathrm{m} \mathrm{B} . \lambda=3.42 \mu \mathrm{m} \mathrm{C}$. $\lambda=3.84 \mu \mathrm{m}$ D. $\lambda=4.54 \mu \mathrm{m}$. The side colorbar in each image indicates the transmittance values.

solid state is constant despite the slight thickness variation of the medium, whereas the sample in the liquid state is more homogeneous with a constant transmission value that is $\approx 2$ times higher than the one for the solid state. An increase in the spectral properties between $36^{\circ} \mathrm{C}$ and $40^{\circ} \mathrm{C}$ is clearly distinguished and confirms that the phase transition occurs in this temperature range. 


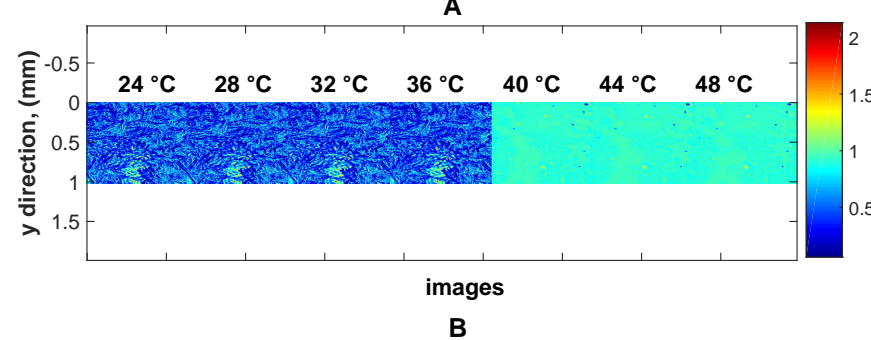

B

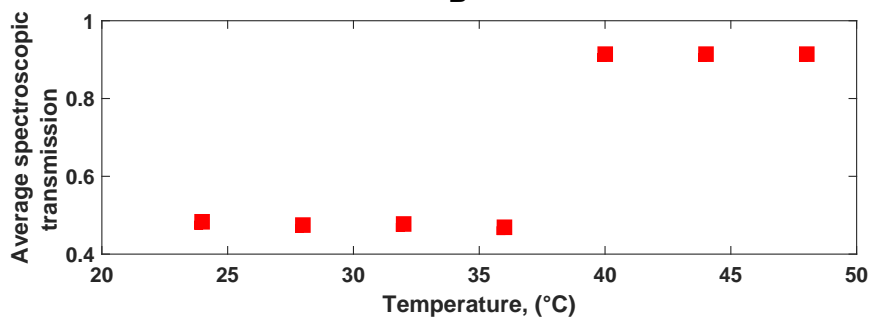

Figure 8: A. Images of the integral of the transmittance spectral images over the characteristic peak (Gaussian distribution around $3.4 \mu \mathrm{m}$ ) at each temperature. The side colorbar indicates the transmittance values. B. The previous images of the integral of the transmittance were averaged along the $\mathrm{x}$ and $\mathrm{y}$ directions, and the obtained value is plotted as a function of the imposed temperature.

\subsection{Thermal imaging: proper emission}

The IR camera and the adapted optics allow us to simultaneously measure the microscopic temperature through the proper emission of the analyzed sample. Here, the observed thermal scene is recorded (when the chopper is closed) for all the imposed temperatures when a steady state is reached (at each plateau, as shown on the ramp in figure 2). The measured signal at each temperature is depicted in Figure 9.A. These images verify that the imposed temperature is spatially homogeneous.

Moreover, in Figure 9.B, the relation between the imposed temperature $\left(\mathrm{T},{ }^{\circ} \mathrm{C}\right.$, given by the thermocouple inside the heat stage) and the measured temperature intensity (DL, Digital Level) by the IR camera is shown. Using this representation, it is possible to demonstrate the polynomial dependence of the DL according to the imposed temperature, particularly for the highest temperatures (after the phase change). However, before the phase change, the DL varied linearly with the absolute temperature, which is due to the low integration time of the camera and to the limited temperature range. This produces a "linear" dependence between the radiative flux (measured temperature
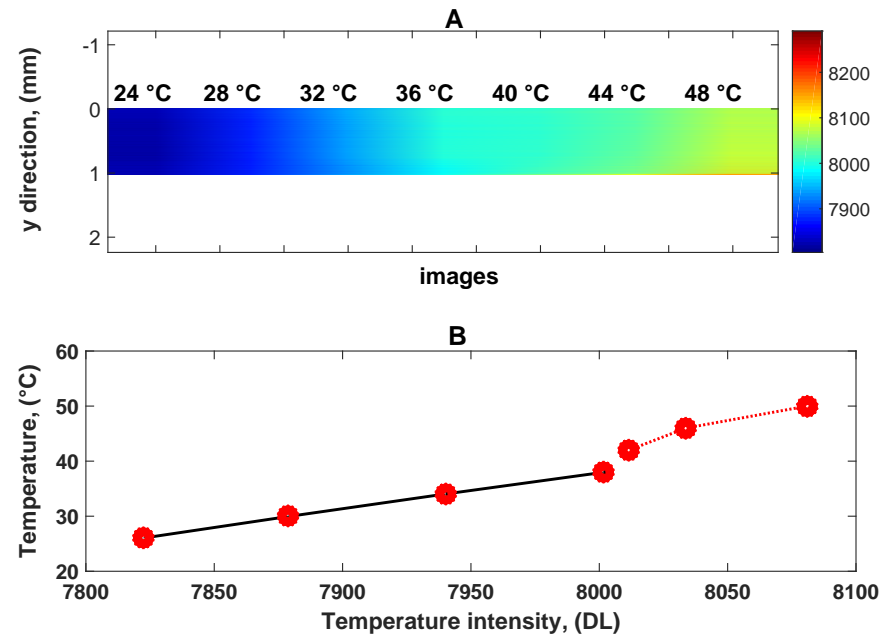

Figure 9: A. Image of the thermal scene measured using IR micro-scale thermography of the paraffin sample at each temperature. The side colorbar indicates the temperature intensity digital level. B Relation between the imposed temperature $\left(\mathrm{T},{ }^{\circ} \mathrm{C}\right.$, given by the thermal probe inside the heat stage) and the measured temperature intensity (DL, Digital Level, from the images in figure A). Two regimes are observed before and after the phase change.

in proper emission) and the one given by the thermal probe in the heat stage. This plotted relation establish a calibration curve to relate the measured temperature intensity and the imposed temperature. Finally, by applying the polynomial calibration to the raw DL data, we retrieved the absolute temperature of the measured field, as shown in Figure 10.

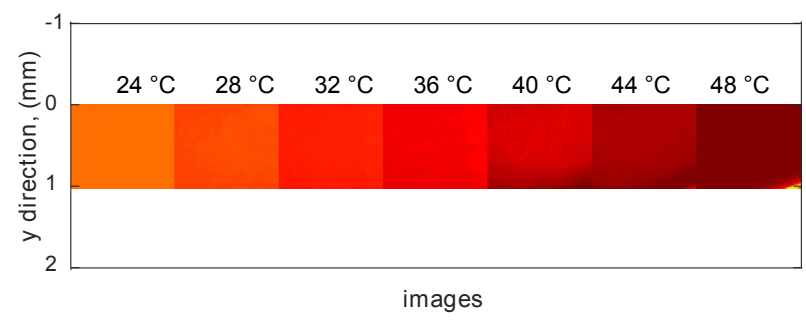

Figure 10: Image of the thermal scene measured using IR micro-scale thermography of the paraffin sample at each temperature after applying the polynomial calibration shown in Figure 9.B to the raw DL data. The absolute temperature in Celsius is indicated.

\section{Conclusions and Perspectives}

In this paper, simultaneous microscopic measurements of thermal and spectroscopic fields of a phase change material, paraffin wax, are reported. The combination of the latest tech- 
nological advances in IR cameras, monochromators and optics were instrumental to achieving the fine metrology to perform the micro-scale thermography and spectroscopy. Because the instrument performs simultaneous measurements, a processing method based on two-image acquisition was proposed to separate the spectroscopic information from the thermal information. In this first study, for validation purposes, only the steady states (plateau of the ramp) in the solid and liquid phase were reported. Thus, paraffin wax spectroscopy at different temperatures was performed, and this analysis reveals that paraffin wax exhibits a higher transmittance in the liquid state than in the solid state. Additionally, imaging spectroscopy reveals that in the solid or liquid state, the paraffin wax transmittance average value is constant, whereas the transmittance value is different for both states. The variation in the spectral properties between $36^{\circ} \mathrm{C}$ and $40^{\circ} \mathrm{C}$ is clearly distinguishable and confirms that the phase transition is in this temperature range. Therefore, thermal imaging enables us to observe the thermal scene for all the imposed temperatures when the steady state is reached and allows us to verify that the imposed temperature is spatially homogeneous. Because of this information, the relation between the imposed temperature (given by the thermal probe) and the measured temperature intensity (DL) by the IR camera is established and allows us to develop a polynomial calibration to retrieve the absolute temperature value of the measured thermal field.

Many perspectives are being considered and are in progress. First, we are studying the paraffin wax phase change process that occurs at the transient state in the temperature range of the phase change. The released heat will be measured in parallel with the estimate of the enthalpy involved during the dynamic process. This work shows that there is no obstacle to studying transient systems by applying the proposed IR methodology and using thermal calibration to perform quantitative measure- ments. This technique is valuable for the structural analysis of materials at the micro-scale. The most important task that remains is to apply this technique to monitor more complex and ultrafast phenomena, such as phase changes, kinetics of chemical reactions, biological systems, and many other phenomena, related to the simultaneous variations of both temperature and concentration.

\section{Acknowledgments}

The authors gratefully acknowledge Bordeaux International Support (BIS)-Japan IDEX founding, and J.M. acknowledges the support of JSPS KAKENHI Grant No. 25420752. This work was partly supported by the Precise Measurement Technology Promotion Foundation(PMTP-F)

\section{References}

[1] A. Mialdun, V. Yasnou, V. Shevtsova, A. Königer, W. Köhler, D. A. de Mezquia, M. Bou-Ali, A comprehensive study of diffusion, thermodiffusion, and soret coefficients of water-isopropanol mixtures, The Journal of chemical physics 136 (2012) 244512.

[2] A. Florent, P. Jean-Franois, G. Emilien, F. Laurent, D. Aurlie, C. Vincent, Estimation of kinetic parameters and diffusion coefficients for the transesterification of triolein with methanol on a solid znal2o4 catalyst, Chemical Engineering Journal (2015) http://dx.doi.org/10.1016/j.cej.2015.07.075.

[3] T. Aillet, K. Loubière, L. Prat, O. Dechy-Cabaret, Impact of the diffusion limitation in microphotoreactors, AIChE Journal 61 (2015) 1284-1299.

[4] G. Fang, H. Li, F. Yang, X. Liu, S. Wu, Preparation and characterization of nano-encapsulated n-tetradecane as phase change material for thermal energy storage, Chemical Engineering Journal 153 (2009) 217-221.

[5] B. He, V. Martin, F. Setterwall, Phase transition temperature ranges and storage density of paraffin wax phase change materials, Energy 29 (2004) 1785-1804.

[6] M. Romano, C. Pradere, F. Sarrazin, J. Toutain, J. Batsale, Enthalpy, kinetics and mixing characterization in droplet-flow millifluidic device by infrared thermography, Chemical Engineering Journal 273 (2015) 325332.

[7] T. Dang, T. Kolkman, F. Gallucci, M. van Sint Annaland, Development of a novel infrared technique for instantaneous, whole-field, non invasive 
gas concentration measurements in gas-solid fluidized beds, Chemical Engineering Journal 219 (2013) 545-557.

[8] M. A. Rahman, Q. Galand, M. Soliman, S. Van Vaerenbergh, M. Saghir, Measurement of refractive indices of binary mixtures using digital interferometry and multi-wavelength abbemat refractometer, Optics and lasers in engineering 51 (2013) 503-513.

[9] M. N. An tSaoir, D. L. A. Fernandes, M. McMaster, K. Kitagawa, C. Hardacre, F. Aiouache, Transient distributions of composition and temperature in a gas-solid packed bed reactor by near-infrared tomography, Chemical Engineering Journal 189 (2012) 383-392.

[10] M. Bessou, B. Chassagne, J.-P. Caumes, C. Pradere, P. Maire, M. Tondusson, E. Abraham, Three-dimensional terahertz computed tomography of human bones, Applied optics 51 (2012) 6738-6744.

[11] J. Morikawa, E. Hayakawa, T. Hashimoto, R. Buividas, S. Juodkazis, Thermal imaging of a heat transport in regions structured by femtosecond laser, Optics express 19 (2011) 20542-20550.

[12] M. Romano, C. Ndiaye, A. Duphil, A. Sommier, J. Morikawa, J. Mascetti, J. Batsale, L. Servant, C. Pradere, Fast infrared imaging spectroscopy technique (fiist), Infrared Physics \& Technology 68 (2015) 152-158. 